\title{
Approximation by First-Order Linear Differential Equations with an Initial Condition
}

\author{
Jaiok Roh ${ }^{1}$ and Soon-Mo Jung ${ }^{2}$ \\ ${ }^{1}$ Department of Mathematics, Hallym University, Chuncheon, Gangwon-do 24252, Republic of Korea \\ ${ }^{2}$ Mathematics Section, College of Science and Technology, Hongik University, Sejong 30016, Republic of Korea \\ Correspondence should be addressed to Soon-Mo Jung; smjung@hongik.ac.kr
}

Received 10 May 2016; Accepted 25 July 2016

Academic Editor: Seppo Hassi

Copyright (C) 2016 J. Roh and S.-M. Jung. This is an open access article distributed under the Creative Commons Attribution License, which permits unrestricted use, distribution, and reproduction in any medium, provided the original work is properly cited.

We will consider a continuously differentiable function $y: I \rightarrow \mathbb{R}$ satisfying the inequality $\left|p(t) y^{\prime}(t)-q(t) y(t)-r(t)\right| \leq \varepsilon$ for all $t \in I$ and $\left|y\left(t_{0}\right)-\alpha\right| \leq \delta$ for some $t_{0} \in I$ and some $\alpha \in \mathbb{R}$. Then we will approximate $y$ by a solution $z$ of the linear equation $p(t) z^{\prime}(t)-q(t) z(t)-r(t)=0$ with $z\left(t_{0}\right)=\alpha$.

\section{Introduction}

The question concerning the stability of functional equations has been originally raised by Ulam [1]: given a metric group $(G, \cdot, d)$, a positive number $\varepsilon$, and a function $f: G \rightarrow G$ which satisfies inequality $d(f(x y), f(x) f(y)) \leq \varepsilon$ for all $x, y \in G$, does there exist homomorphism $a: G \rightarrow G$ and a constant $\delta$ depending only on $G$ and $\varepsilon$ such that $d(a(x), f(x)) \leq \delta$ for all $x \in G$ ?

If the answer to this question is affirmative, the functional equation $a(x y)=a(x) a(y)$ is said to be stable. A first answer to this question was given by Hyers [2] in 1941 who proved that the Cauchy additive equation is stable in Banach spaces. In general, a functional equation is said to be stable in the sense of Hyers and Ulam (or the equation has the HyersUlam stability) if, for each solution to the perturbed equation, there exists a solution to the equation that differs from the solution to the perturbed equation with a small error. We refer the reader to [3-8] for the exact definition of Hyers-Ulam stability.

Usually the experiment (or the observed) data do not exactly coincide with theoretical ones. We may express natural phenomena by use of equations but because of the errors due to measurement or observance the actual experiment data can almost always be a little bit off the expectations. If we would use inequalities instead of equalities to explain natural phenomena, then these errors could be absorbed into the solutions of inequalities; that is, those errors would be no more errors.

There is another way to explain the Hyers-Ulam stability. Let us consider a closed system which can be explained by the first-order linear differential equation, namely, $y^{\prime}(t)=\lambda y(t)$. The past, present, and future of this system are completely determined if we know the general solution and an initial condition of that differential equation. So we can say that this system is "predictable." Sometimes, because of the disturbances (or noises) of the outside, the system may not be determined by $y^{\prime}(t)=\lambda y(t)$ but can only be explained by an inequality like $\left|y^{\prime}(t)-\lambda y(t)\right| \leq \varepsilon$. Then it is impossible to predict the exact future of the disturbed system.

Even though the system is not predictable exactly because of outside disturbances, we say the differential equation $y^{\prime}(t)=\lambda y(t)$ has the Hyers-Ulam stability if the "real" future of the system follows the solution of $y^{\prime}(t)=\lambda y(t)$ with a bounded error. But if the error bound is "too big," we say that differential equation $y^{\prime}(t)=\lambda y(t)$ does not have the HyersUlam stability. Resonance is the case. Considering this point of view, the Hyers-Ulam stability (of differential equations) is fundamental (see [9]).

A generalization of Ulam's problem was recently proposed by replacing functional equations with differential equations. Obloza seems to be the first author who has investigated the Hyers-Ulam stability of linear differential 
equations (see $[10,11]$ ). Thereafter, Alsina and Ger proved the Hyers-Ulam stability of the linear differential equations: indeed, they proved in [12] that if a differentiable function $f: I \rightarrow \mathbb{R}$ is a solution of the differential inequality $\mid y^{\prime}(t)-$ $y(t) \mid \leq \varepsilon$, where $I$ is an open subinterval of $\mathbb{R}$, then there exists a solution $f_{0}: I \rightarrow \mathbb{R}$ of the differential equation $y^{\prime}(t)=y(t)$ such that $\left|f(t)-f_{0}(t)\right| \leq 3 \varepsilon$ for any $t \in I$.

The above result by Alsina and Ger was generalized by Miura et al. [13], by Miura et al. [14], and also by Takahasi et al. $[15,16]$. They proved that the Hyers-Ulam stability holds true for the Banach space-valued differential equation $y^{\prime}=\lambda y$.

Miura et al. [14] proved the Hyers-Ulam stability of the first-order linear differential equation $y^{\prime}(t)+g(t) y(t)=0$, where $g$ is a continuous function, while Jung [17] proved the Hyers-Ulam stability of the differential equation of the form $\varphi(t) y^{\prime}(t)=y(t)$. Furthermore, results of Hyers-Ulam stability of first-order linear differential equation have been generalized by Miura et al. [18] and also by Takahasi et al. [16]. They dealt with the nonhomogeneous linear differential equation of the form $y^{\prime}(t)+g(t) y(t)+h(t)=0$.

Wang et al. [19] used the method of integrating factor to prove the Hyers-Ulam stability of the nonhomogeneous linear differential equation of the form

$$
p(t) y^{\prime}(t)-q(t) y(t)-r(t)=0,
$$

where $t \in I=(a, b)$ with $-\infty \leq a<b \leq \infty$.

Theorem 1 (see [19]). Let $p, q, r: I \rightarrow \mathbb{R}$ be continuous functions such that $p(t) \neq 0$ and

$$
|q(t)| \geq \mu
$$

for all $t \in I$ and some constant $\mu>0$. Given a constant $\varepsilon>0$, if a continuously differentiable function $y: I \rightarrow \mathbb{R}$ satisfies the differential inequality

$$
\left|p(t) y^{\prime}(t)-q(t) y(t)-r(t)\right| \leq \varepsilon
$$

for all $t \in I$, then there exists a solution $z: I \rightarrow \mathbb{R}$ of the differential equation (1) such that

$$
|y(t)-z(t)| \leq \varepsilon\left(2 \exp \left\{\left|\int_{a}^{b} \frac{q(s)}{p(s)} d s\right|\right\}-1\right)
$$

for all $t \in I$, where $z: I \rightarrow \mathbb{R}$ is a function defined by

$$
\begin{aligned}
& z(t)=\exp \left\{\int_{a}^{t} \frac{q(s)}{p(s)} d s\right\} \\
& \cdot\left(\left(y\left(b_{1}\right)-\varepsilon\right) \exp \left\{-\int_{a}^{b_{1}} \frac{q(s)}{p(s)} d s\right\}\right. \\
& \left.-\int_{t}^{b_{1}} \frac{r(s)}{p(s)} \exp \left\{-\int_{a}^{s} \frac{q(u)}{p(u)} d u\right\} d s\right) .
\end{aligned}
$$

We wondered if a stability of (1) can be proven without the condition $|q(t)| \geq \mu$ in Theorem 1 and whether the error estimation of Theorem 1 can be improved or not.
In this paper, we are going to prove the Hyers-Ulam stability of the differential equation (1) with an initial condition and then compare the stability results of (1) with and without the condition $|q(t)| \geq \mu$. And also we are going to generalize the stability result of (1) by replacing the bounded difference with a general control function.

Throughout this paper, let $I$ denote an open interval $(a, b)$ with $-\infty \leq a<b \leq \infty$.

\section{Hyers-Ulam Stability without (2)}

In the following theorem, we shall prove the Hyers-Ulam stability of the differential equation (1) with an initial condition when condition (2) is excluded, while Wang et al. proved the Hyers-Ulam stability of (1) with condition (2) (see Theorem 1 or [19]).

Theorem 2. Let $p, q, r: I \rightarrow \mathbb{R}$ be continuous functions such that

(i) $p(t) \neq 0$ for all $t \in I$;

(ii) $\int_{a}^{t}(q(s) / p(s)) d s$ exists for every $t \in I$.

Given constants $\varepsilon>0$ and $\delta>0$, if a continuously differentiable function $y: I \rightarrow \mathbb{R}$ satisfies the differential inequality

$$
\left|p(t) y^{\prime}(t)-q(t) y(t)-r(t)\right| \leq \varepsilon
$$

for all $t \in I$ with

$$
\left|y\left(t_{0}\right)-\alpha\right| \leq \delta
$$

for some $t_{0} \in I$ and $\alpha \in \mathbb{R}$, then there exists a solution $z: I \rightarrow$ $\mathbb{R}$ of the differential equation (1) with $z\left(t_{0}\right)=\alpha$ such that

$$
\begin{aligned}
& |y(t)-z(t)| \leq \varepsilon \exp \left\{\int_{a}^{t} \frac{q(s)}{p(s)} d s\right\} \\
& \cdot\left|\int_{t_{0}}^{t} \frac{1}{p(s)} \exp \left\{-\int_{a}^{s} \frac{q(u)}{p(u)} d u\right\} d s\right|+\delta \\
& \cdot \exp \left\{\int_{t_{0}}^{t} \frac{q(s)}{p(s)} d s\right\}
\end{aligned}
$$

for all $t \in I$.

Proof. Assume that $p(t)>0$ for all $t \in I$. In view of (6), we have

$$
-\varepsilon \leq p(t) y^{\prime}(t)-q(t) y(t)-r(t) \leq \varepsilon
$$


for any $t \in I$. Multiplying the formula by (1/ $p(t)) \exp \left\{-\int_{a}^{t}(q(s) / p(s)) d s\right\}$, we get

$$
\begin{aligned}
& -\frac{\varepsilon}{p(t)} \exp \left\{-\int_{a}^{t} \frac{q(s)}{p(s)} d s\right\} \\
& \leq \exp \left\{-\int_{a}^{t} \frac{q(s)}{p(s)} d s\right\} y^{\prime}(t) \\
& -\frac{q(t)}{p(t)} \exp \left\{-\int_{a}^{t} \frac{q(s)}{p(s)} d s\right\} y(t) \\
& -\frac{r(t)}{p(t)} \exp \left\{-\int_{a}^{t} \frac{q(s)}{p(s)} d s\right\} \\
& \leq \frac{\varepsilon}{p(t)} \exp \left\{-\int_{a}^{t} \frac{q(s)}{p(s)} d s\right\}
\end{aligned}
$$

for all $t \in I$.

Integrating each term of (10) from $t$ to $t_{0}$, we have

$$
\begin{array}{r}
-\varepsilon\left|\int_{t}^{t_{0}} \frac{1}{p(s)} \exp \left\{-\int_{a}^{s} \frac{q(u)}{p(u)} d u\right\} d s\right| \\
\leq \exp \left\{-\int_{a}^{t_{0}} \frac{q(s)}{p(s)} d s\right\} y\left(t_{0}\right) \\
-\exp \left\{-\int_{a}^{t} \frac{q(s)}{p(s)} d s\right\} y(t) \\
-\int_{t}^{t_{0}} \frac{r(s)}{p(s)} \exp \left\{-\int_{a}^{s} \frac{q(u)}{p(u)} d u\right\} d s \\
\leq \varepsilon\left|\int_{t}^{t_{0}} \frac{1}{p(s)} \exp \left\{-\int_{a}^{s} \frac{q(u)}{p(u)} d u\right\} d s\right|
\end{array}
$$

for all $t \in I$. Then

$$
\begin{array}{r}
-\varepsilon\left|\int_{t}^{t_{0}} \frac{1}{p(s)} \exp \left\{-\int_{a}^{s} \frac{q(u)}{p(u)} d u\right\} d s\right| \\
\leq \exp \left\{-\int_{a}^{t_{0}} \frac{q(s)}{p(s)} d s\right\}\left(y\left(t_{0}\right)-\alpha\right) \\
+\alpha \exp \left\{-\int_{a}^{t_{0}} \frac{q(s)}{p(s)} d s\right\} \\
-\int_{t}^{t_{0}} \frac{r(s)}{p(s)} \exp \left\{-\int_{a}^{s} \frac{q(u)}{p(u)} d u\right\} d s \\
\quad-\exp \left\{-\int_{a}^{t} \frac{q(s)}{p(s)} d s\right\} y(t) \\
\leq \varepsilon\left|\int_{t}^{t_{0}} \frac{1}{p(s)} \exp \left\{-\int_{a}^{s} \frac{q(u)}{p(u)} d u\right\} d s\right|
\end{array}
$$

for all $t \in I$.
It follows from (7) and (12) that

$$
\begin{aligned}
& -\varepsilon\left|\int_{t}^{t_{0}} \frac{1}{p(s)} \exp \left\{-\int_{a}^{s} \frac{q(u)}{p(u)} d u\right\} d s\right| \\
& -\delta \exp \left\{-\int_{a}^{t_{0}} \frac{q(s)}{p(s)} d s\right\} \leq \alpha \exp \left\{-\int_{a}^{t_{0}} \frac{q(s)}{p(s)} d s\right\} \\
& -\int_{t}^{t_{0}} \frac{r(s)}{p(s)} \exp \left\{-\int_{a}^{s} \frac{q(u)}{p(u)} d u\right\} d s \\
& -\exp \left\{-\int_{a}^{t} \frac{q(s)}{p(s)} d s\right\} y(t) \\
& \leq \varepsilon\left|\int_{t}^{t_{0}} \frac{1}{p(s)} \exp \left\{-\int_{a}^{s} \frac{q(u)}{p(u)} d u\right\} d s\right| \\
& +\delta \exp \left\{-\int_{a}^{t_{0}} \frac{q(s)}{p(s)} d s\right\}
\end{aligned}
$$

for all $t \in I$. Multiplying (13) by $\exp \left\{\int_{a}^{t}(q(s) / p(s)) d s\right\}$, we have

$$
\begin{aligned}
& \mid \alpha \exp \left\{-\int_{t}^{t_{0}} \frac{q(s)}{p(s)} d s\right\}-\exp \left\{\int_{a}^{t} \frac{q(s)}{p(s)} d s\right\} \\
& \cdot \int_{t}^{t_{0}} \frac{r(s)}{p(s)} \exp \left\{-\int_{a}^{s} \frac{q(u)}{p(u)} d u\right\} d s-y(t) \mid \leq \varepsilon \\
& \cdot \exp \left\{\int_{a}^{t} \frac{q(s)}{p(s)} d s\right\} \\
& \cdot\left|\int_{t}^{t_{0}} \frac{1}{p(s)} \exp \left\{-\int_{a}^{s} \frac{q(u)}{p(u)} d u\right\} d s\right|+\delta \\
& \cdot \exp \left\{-\int_{t}^{t_{0}} \frac{q(s)}{p(s)} d s\right\}
\end{aligned}
$$

for all $t \in I$.

If we define a function $z: I \rightarrow \mathbb{R}$ by

$$
\begin{gathered}
z(t):=\alpha \exp \left\{\int_{t_{0}}^{t} \frac{q(s)}{p(s)} d s\right\}+\exp \left\{\int_{a}^{t} \frac{q(s)}{p(s)} d s\right\} \\
\cdot \int_{t_{0}}^{t} \frac{r(s)}{p(s)} \exp \left\{-\int_{a}^{s} \frac{q(u)}{p(u)} d u\right\} d s
\end{gathered}
$$

for all $t \in I$, then $z$ is a solution of the differential equation (1) with $z\left(t_{0}\right)=\alpha$. Moreover, in view of (14), we get

$$
\begin{aligned}
|y(t)-z(t)| \leq & \varepsilon \exp \left\{\int_{a}^{t} \frac{q(s)}{p(s)} d s\right\} \\
& \cdot\left|\int_{t_{0}}^{t} \frac{1}{p(s)} \exp \left\{-\int_{a}^{s} \frac{q(u)}{p(u)} d u\right\} d s\right| \\
& +\delta \exp \left\{\int_{t_{0}}^{t} \frac{q(s)}{p(s)} d s\right\}
\end{aligned}
$$

for all $t \in I$.

By an argument similar to the above, for the case when $p(t)<0$ for all $t \in I$, we get the same result. This completes the proof of this theorem. 
Corollary 3. Let $p, q, r: I \rightarrow \mathbb{R}$ be continuous functions such that

(i) $p(t) \neq 0$ for all $t \in I$;

(ii) $q(t) / p(t)>0$ for all $t \in I$;

(iii) $\int_{a}^{t}(q(s) / p(s)) d s$ exists for all $t \in I$.

Moreover, assume that there exists $t_{0} \in I$ such that

(iv) $\int_{t_{0}}^{b}(q(s) / p(s)) d s \leq \ln 2$.

Given constants $\varepsilon>0$ and $\delta>0$, if a continuously differentiable function $y: I \rightarrow \mathbb{R}$ satisfies the differential inequality (6) with (7) for all $t \in I$ and for some $\alpha \in \mathbb{R}$, then there exists a solution $z: I \rightarrow \mathbb{R}$ of the differential equation (1) with $z\left(t_{0}\right)=\alpha$ such that

$$
|y(t)-z(t)| \leq 2 \varepsilon\left|\int_{t_{0}}^{t} \frac{1}{p(s)} d s\right|+2 \delta
$$

for all $t \in I$.

Proof. In view of (ii), (iii), and (iv), it is not difficult to show that

$$
0 \leq \exp \left\{\int_{s}^{t} \frac{q(u)}{p(u)} d u\right\} \leq 2
$$

for all $s, t \in I$. Hence, it follows from Theorem 2 that

$$
\begin{aligned}
& |y(t)-z(t)| \leq \varepsilon \exp \left\{\int_{a}^{t} \frac{q(s)}{p(s)} d s\right\} \\
& \cdot\left|\int_{t_{0}}^{t} \frac{1}{p(s)} \exp \left\{-\int_{a}^{s} \frac{q(u)}{p(u)} d u\right\} d s\right|+\delta \\
& \cdot \exp \left\{\int_{t_{0}}^{t} \frac{q(s)}{p(s)} d s\right\} \\
& =\varepsilon\left|\int_{t_{0}}^{t} \frac{1}{p(s)} \exp \left\{\int_{s}^{t} \frac{q(u)}{p(u)} d u\right\} d s\right|+\delta \\
& \cdot \exp \left\{\int_{t_{0}}^{t} \frac{q(s)}{p(s)} d s\right\} \leq 2 \varepsilon\left|\int_{t_{0}}^{t} \frac{1}{p(s)} d s\right|+2 \delta
\end{aligned}
$$

for all $t \in I$.

In the next theorem, we are going to generalize the stability result of the differential equation (1) by a general control function $\varphi(t)$.

Theorem 4. Let $p, q, r: I \rightarrow \mathbb{R}$ be continuous functions such that

(i) $p(t) \neq 0$ for all $t \in I$;

(ii) $\int_{a}^{t}(q(s) / p(s)) d s$ exists for any $t \in I$.

Suppose $\varphi: I \rightarrow[0, \infty)$ is a continuous function. Given a constant $\delta>0$, if a continuously differentiable function $y$ : $I \rightarrow \mathbb{R}$ satisfies the differential inequality

$$
\left|p(t) y^{\prime}(t)-q(t) y(t)-r(t)\right| \leq \varphi(t)
$$

for all $t \in I$ with

$$
\left|y\left(t_{0}\right)-\alpha\right| \leq \delta
$$

for some $t_{0} \in I$ and $\alpha \in \mathbb{R}$, then there exists a solution $z: I \rightarrow$ $\mathbb{R}$ of the differential equation (1) with $z\left(t_{0}\right)=\alpha$ such that

$$
\begin{aligned}
|y(t)-z(t)| \leq & \exp \left\{\int_{a}^{t} \frac{q(s)}{p(s)} d s\right\} \\
& \cdot\left|\int_{t_{0}}^{t} \frac{\varphi(s)}{p(s)} \exp \left\{-\int_{a}^{s} \frac{q(u)}{p(u)} d u\right\} d s\right| \\
& +\delta \exp \left\{\int_{t_{0}}^{t} \frac{q(s)}{p(s)} d s\right\}
\end{aligned}
$$

for all $t \in I$.

Proof. Similarly to Theorem 2, we can prove this theorem.

Corollary 5. Let $p, q, r: I \rightarrow \mathbb{R}$ be continuous functions and let $t_{0} \in I$ be given such that

(i) $p(t) \neq 0$ for all $t \in I$;

(ii) $q(t) / p(t)>0$ for all $t \in I$;

(iii) $\int_{a}^{t}(q(s) / p(s)) d s$ exists for all $t \in I$;

(iv) $\int_{t_{0}}^{b}(q(s) / p(s)) d s \leq \ln 2$.

Suppose $\varphi: I \rightarrow[0, \infty)$ is a continuous function. Given a constant $\delta>0$, if a continuously differentiable function $y$ : $I \rightarrow \mathbb{R}$ satisfies the differential inequality (20) with (21) for all $t \in I$ and for some $\alpha \in \mathbb{R}$, then there exists a solution $z: I \rightarrow \mathbb{R}$ of the differential equation (1) with $z\left(t_{0}\right)=\alpha$ such that

$$
|y(t)-z(t)| \leq 2\left|\int_{t_{0}}^{t} \frac{\varphi(s)}{p(s)} d s\right|+2 \delta
$$

for all $t \in I$.

Proof. As in the proof of Corollary 3, by (ii), (iii), and (iv), we can easily show that

$$
0 \leq \exp \left\{\int_{s}^{t} \frac{q(u)}{p(u)} d u\right\} \leq 2
$$

for all $s, t \in I$. Therefore, it follows from Theorem 4 that

$$
\begin{aligned}
& |y(t)-z(t)| \leq \exp \left\{\int_{a}^{t} \frac{q(s)}{p(s)} d s\right\} \\
& \cdot\left|\int_{t_{0}}^{t} \frac{\varphi(s)}{p(s)} \exp \left\{-\int_{a}^{s} \frac{q(u)}{p(u)} d u\right\} d s\right|+\delta \\
& \cdot \exp \left\{\int_{t_{0}}^{t} \frac{q(s)}{p(s)} d s\right\} \\
& =\left|\int_{t_{0}}^{t} \frac{\varphi(s)}{p(s)} \exp \left\{\int_{s}^{t} \frac{q(u)}{p(u)} d u\right\} d s\right|+\delta \\
& \cdot \exp \left\{\int_{t_{0}}^{t} \frac{q(s)}{p(s)} d s\right\} \leq 2\left|\int_{t_{0}}^{t} \frac{\varphi(s)}{p(s)} d s\right|+2 \delta
\end{aligned}
$$

for all $t \in I$. 


\section{Hyers-Ulam Stability with (2)}

In this section, we also assume that $I$ is an open interval $(a, b)$ for $-\infty \leq a<b \leq \infty$ and we prove, in the following theorem, the Hyers-Ulam stability of the differential equation (1) with an initial condition when condition (2) is included.

Theorem 6. Let $p, q, r: I \rightarrow \mathbb{R}$ be continuous functions such that

(i) $p(t) \neq 0$ for all $t \in I$;

(ii) $q(t) / p(t)>0$ for all $t \in I$;

(iii) $\int_{a}^{t}(q(s) / p(s)) d s$ exists for any $t \in I$.

Moreover, assume that there exist $t_{0} \in I$ and a constant $\mu>0$ such that

(iv) $\int_{t_{0}}^{b}(q(s) / p(s)) d s \leq \ln 2$;

(v) $|q(t)| \geq \mu$ for all $t \in I$.

Given constants $\varepsilon>0$ and $\delta>0$, if a continuously differentiable function $y: I \rightarrow \mathbb{R}$ satisfies the differential inequality (6) with (7) for all $t \in I$ and for some $\alpha \in \mathbb{R}$, then there exists a solution $z: I \rightarrow \mathbb{R}$ of the differential equation (1) with $z\left(t_{0}\right)=\alpha$ such that

$$
|y(t)-z(t)| \leq \frac{\varepsilon}{\mu}+2 \delta
$$

for all $t \in I$.

Proof. Assume that $p(t)>0$ and $q(t) \geq \mu>0$ for all $t \in I$. Since $q(t) / \mu \geq 1$ for each $t \in I$, it follows from (10) that

$$
\begin{aligned}
& -\frac{\varepsilon q(t)}{\mu p(t)} \exp \left\{-\int_{a}^{t} \frac{q(s)}{p(s)} d s\right\} \\
& \leq \exp \left\{-\int_{a}^{t} \frac{q(s)}{p(s)} d s\right\} y^{\prime}(t) \\
& -\frac{q(t)}{p(t)} \exp \left\{-\int_{a}^{t} \frac{q(s)}{p(s)} d s\right\} y(t) \\
& -\frac{r(t)}{p(t)} \exp \left\{-\int_{a}^{t} \frac{q(s)}{p(s)} d s\right\} \\
& \leq \frac{\varepsilon q(t)}{\mu p(t)} \exp \left\{-\int_{a}^{t} \frac{q(s)}{p(s)} d s\right\}
\end{aligned}
$$

for all $t \in I$. Since the function $y$ is continuous, we know that $y\left(t_{0}\right)$ is finite. And, for any $t \in I$, by integrating (27) from $t$ to $t_{0}$, we have

$$
\begin{gathered}
-\frac{\varepsilon}{\mu}\left|\exp \left\{-\int_{a}^{t} \frac{q(s)}{p(s)} d s\right\}-\exp \left\{-\int_{a}^{t_{0}} \frac{q(s)}{p(s)} d s\right\}\right| \\
\leq \exp \left\{-\int_{a}^{t_{0}} \frac{q(s)}{p(s)} d s\right\} y\left(t_{0}\right) \\
-\exp \left\{-\int_{a}^{t} \frac{q(s)}{p(s)} d s\right\} y(t)
\end{gathered}
$$

$$
\begin{aligned}
& -\int_{t}^{t_{0}} \frac{r(s)}{p(s)} \exp \left\{-\int_{a}^{s} \frac{q(u)}{p(u)} d u\right\} d s \\
\leq & \frac{\varepsilon}{\mu}\left|\exp \left\{-\int_{a}^{t} \frac{q(s)}{p(s)} d s\right\}-\exp \left\{-\int_{a}^{t_{0}} \frac{q(s)}{p(s)} d s\right\}\right| .
\end{aligned}
$$

Then

$$
\begin{aligned}
-\frac{\varepsilon}{\mu}\left|\exp \left\{-\int_{a}^{t} \frac{q(s)}{p(s)} d s\right\}-\exp \left\{-\int_{a}^{t_{0}} \frac{q(s)}{p(s)} d s\right\}\right| \\
\leq \exp \left\{-\int_{a}^{t_{0}} \frac{q(s)}{p(s)} d s\right\}\left(y\left(t_{0}\right)-\alpha\right) \\
+\alpha \exp \left\{-\int_{a}^{t_{0}} \frac{q(s)}{p(s)} d s\right\} \\
-\exp \left\{-\int_{a}^{t} \frac{q(s)}{p(s)} d s\right\} y(t) \\
-\int_{t}^{t_{0}} \frac{r(s)}{p(s)} \exp \left\{-\int_{a}^{s} \frac{q(u)}{p(u)} d u\right\} d s \\
\leq \frac{\varepsilon}{\mu}\left|\exp \left\{-\int_{a}^{t} \frac{q(s)}{p(s)} d s\right\}-\exp \left\{-\int_{a}^{t_{0}} \frac{q(s)}{p(s)} d s\right\}\right|
\end{aligned}
$$

for each $t \in I$

It follows from (7) and (29) that

$$
\begin{aligned}
& -\frac{\varepsilon}{\mu}\left|\exp \left\{-\int_{a}^{t} \frac{q(s)}{p(s)} d s\right\}-\exp \left\{-\int_{a}^{t_{0}} \frac{q(s)}{p(s)} d s\right\}\right| \\
& -\delta \exp \left\{-\int_{a}^{t_{0}} \frac{q(s)}{p(s)} d s\right\} \leq \alpha \exp \left\{-\int_{a}^{t_{0}} \frac{q(s)}{p(s)} d s\right\} \\
& -\exp \left\{-\int_{a}^{t} \frac{q(s)}{p(s)} d s\right\} y(t) \\
& -\int_{t}^{t_{0}} \frac{r(s)}{p(s)} \exp \left\{-\int_{a}^{s} \frac{q(u)}{p(u)} d u\right\} d s \\
& \leq \frac{\varepsilon}{\mu}\left|\exp \left\{-\int_{a}^{t} \frac{q(s)}{p(s)} d s\right\}-\exp \left\{-\int_{a}^{t_{0}} \frac{q(s)}{p(s)} d s\right\}\right| \\
& +\delta \exp \left\{-\int_{a}^{t_{0}} \frac{q(s)}{p(s)} d s\right\}
\end{aligned}
$$

for all $t \in I$. Multiplying (30) by $\exp \left\{\int_{a}^{t}(q(s) / p(s)) d s\right\}$, we get

$$
\begin{aligned}
& -\frac{\varepsilon}{\mu}\left|1-\exp \left\{-\int_{t}^{t_{0}} \frac{q(s)}{p(s)} d s\right\}\right|-\delta \exp \left\{-\int_{t}^{t_{0}} \frac{q(s)}{p(s)} d s\right\} \\
& \leq \alpha \exp \left\{-\int_{t}^{t_{0}} \frac{q(s)}{p(s)} d s\right\}-y(t) \\
& -\exp \left\{\int_{a}^{t} \frac{q(s)}{p(s)} d s\right\}
\end{aligned}
$$




$$
\begin{aligned}
& \cdot \int_{t}^{t_{0}} \frac{r(s)}{p(s)} \exp \left\{-\int_{a}^{s} \frac{q(u)}{p(u)} d u\right\} d s \\
& \leq \frac{\varepsilon}{\mu}\left|1-\exp \left\{-\int_{t}^{t_{0}} \frac{q(s)}{p(s)} d s\right\}\right|+\delta \\
& \cdot \exp \left\{-\int_{t}^{t_{0}} \frac{q(s)}{p(s)} d s\right\}
\end{aligned}
$$

for all $t \in I$.

It obviously follows from (ii), (iii), and (iv) that

$$
\begin{aligned}
-\int_{t}^{t_{0}} \frac{q(s)}{p(s)} d s & \leq \ln 2 \\
\text { or } 0 & \leq \exp \left\{-\int_{t}^{t_{0}} \frac{q(s)}{p(s)} d s\right\} \leq 2
\end{aligned}
$$

for all $t \in I$. Hence, by (31), we obtain

$$
\begin{aligned}
& -\frac{\varepsilon}{\mu}-2 \delta \leq \alpha \exp \left\{\int_{t_{0}}^{t} \frac{q(s)}{p(s)} d s\right\}-\exp \left\{\int_{a}^{t} \frac{q(s)}{p(s)} d s\right\} \\
& \quad \cdot \int_{t}^{t_{0}} \frac{r(s)}{p(s)} \exp \left\{-\int_{a}^{s} \frac{q(u)}{p(u)} d u\right\} d s-y(t) \leq \frac{\varepsilon}{\mu} \\
& \quad+2 \delta
\end{aligned}
$$

for all $t \in I$. So, we have

$$
|y(t)-z(t)| \leq \frac{\varepsilon}{\mu}+2 \delta,
$$

where

$$
\begin{aligned}
z(t):= & \alpha \exp \left\{\int_{t_{0}}^{t} \frac{q(s)}{p(s)} d s\right\}+\exp \left\{\int_{a}^{t} \frac{q(s)}{p(s)} d s\right\} \\
& \cdot \int_{t_{0}}^{t} \frac{r(s)}{p(s)} \exp \left\{-\int_{a}^{s} \frac{q(u)}{p(u)} d u\right\} d s
\end{aligned}
$$

for all $t \in I$. Obviously, $z$ is solution of (1) with $z\left(t_{0}\right)=\alpha$.

By an argument similar to the above, for the case when $p(t)<0$ for every $t \in I$, we can prove our assertion.

We remark that if $q(t) / p(t)>0$, for all $t \in I$, $\int_{a}^{b}(q(s) / p(s)) d s \leq \ln 2$ and $\mu \geq 1$, and if $\delta \leq \varepsilon$, then it seems more feasible that our result (Theorem 6 ) is better than the result of Wang et al. (Theorem 1) as we see in the following example.

Example 7. For any $\varepsilon>0$ and for some $0<a<1$, let $I=$ $(a, \infty), p(t)=3 t^{2}, q(t)=2, r(t) \equiv 0, t_{0}=1, \mu=2$, and $\delta=\varepsilon$. If a continuously differentiable function $y: I \rightarrow \mathbb{R}$ satisfies the differential inequality

$$
\begin{array}{r}
\left|3 t^{2} y^{\prime}(t)-2 y(t)\right| \leq \varepsilon \\
|y(1)-\alpha| \leq \varepsilon
\end{array}
$$

for all $t \in I$ and for some real number $\alpha$, then Theorem 6 implies that there exists a continuously differentiable function $z: I \rightarrow \mathbb{R}$ such that

$$
\begin{aligned}
3 t^{2} z^{\prime}(t)-2 z(t) & =0 \quad \text { with } z(1)=\alpha, \\
|y(t)-z(t)| & \leq \frac{5}{2} \varepsilon
\end{aligned}
$$

for all $t \in I$, where $z$ is uniquely determined as

$$
z(t)=\alpha \exp \left\{-\frac{2}{3}\left(\frac{1}{t}-1\right)\right\}
$$

According to Theorem 1, we have

$$
\begin{aligned}
|y(t)-z(t)| \leq\left(2 \exp \left\{\frac{2}{3 a}\right\}-1\right) \varepsilon \rightarrow & \infty, \\
& \text { as } a \longrightarrow 0^{+},
\end{aligned}
$$

instead of (37), which implies that Theorem 6 is better than Theorem 1 for small values of $a$.

Corollary 8. Let $p, q, r: I \rightarrow \mathbb{R}$ be continuous functions such that

(i) $p(t) \neq 0$ for all $t \in I$;

(ii) $q(t) / p(t)<0$ for all $t \in I$;

(iii) $\int_{a}^{t}(q(s) / p(s)) d s$ exists for any $t \in I$.

Moreover, assume that there exist $t_{0} \in I$ and a constant $\mu>0$ such that

(iv) $-\int_{a}^{t_{0}}(q(s) / p(s)) d s \leq \ln 2$;

(v) $|q(t)| \geq \mu$ for all $t \in I$.

Given constants $\varepsilon>0$ and $\delta>0$, if a continuously differentiable function $y: I \rightarrow \mathbb{R}$ satisfies the differential inequality (6) with (7) for all $t \in I$ and for some $\alpha \in \mathbb{R}$, then there exists a solution $z: I \rightarrow \mathbb{R}$ of the differential equation (1) with $z\left(t_{0}\right)=\alpha$ such that

$$
|y(t)-z(t)| \leq \frac{\varepsilon}{\mu}+2 \delta
$$

for all $t \in I$.

\section{Conclusion}

In this paper, we proved the Hyers-Ulam stability of the linear differential equation $p(t) y^{\prime}(t)-q(t) y(t)-r(t)=0$ with an initial condition, $y\left(t_{0}\right)=\alpha$, when the condition, $|q(t)| \geq \mu$, for all $t \in I$ and some constant $\mu>0$, is excluded or included. Due to the hypotheses of Corollary 3 or Theorem 6 , we have

$$
\begin{aligned}
\mu\left|\int_{t_{0}}^{b} \frac{1}{p(s)} d s\right| & =\mu \int_{t_{0}}^{b} \frac{1}{|p(s)|} d s \leq \int_{t_{0}}^{b} \frac{|q(s)|}{|p(s)|} d s \\
& =\int_{t_{0}}^{b} \frac{q(s)}{p(s)} d s \leq \ln 2 ;
\end{aligned}
$$


that is,

$$
\left|\int_{t_{0}}^{b} \frac{1}{p(s)} d s\right| \leq \frac{\ln 2}{\mu} .
$$

We now define a subinterval $I^{+}, I^{0}$, and $I^{-}$by

$$
\begin{aligned}
& I^{+}=\left\{t \in I:\left|\int_{t_{0}}^{t} \frac{1}{p(s)} d s\right|<\frac{1}{2 \mu}\right\}, \\
& I^{0}=\left\{t \in I:\left|\int_{t_{0}}^{t} \frac{1}{p(s)} d s\right|=\frac{1}{2 \mu}\right\}, \\
& I^{-}=\left\{t \in I:\left|\int_{t_{0}}^{t} \frac{1}{p(s)} d s\right|>\frac{1}{2 \mu}\right\} .
\end{aligned}
$$

If $t \in I^{+}$, then we get

$$
\begin{aligned}
& \left|\int_{t_{0}}^{t} \frac{1}{p(s)} d s\right|<\frac{1}{2 \mu} \\
& \quad \text { iff } 2 \varepsilon\left|\int_{t_{0}}^{t} \frac{1}{p(s)} d s\right|+2 \delta<\frac{\varepsilon}{\mu}+2 \delta .
\end{aligned}
$$

This relation implies that if $t \in I^{+}$, then the result of Corollary 3 is better than that of Theorem 6, while if $t \in I^{-}$, then the result of Corollary 3 is worse than that of Theorem 6. Thus, roughly speaking, if $|p(t)|$ is so "large" that $\left|\int_{a}^{b}(1 / p(s)) d s\right|<1 / 2 \mu$ holds, then the result of Corollary 3 is better than that of Theorem 6 .

\section{Competing Interests}

The authors declare that they have no competing interests.

\section{Authors' Contributions}

All authors read and approved the final paper.

\section{Acknowledgments}

This research was supported by Basic Science Research Program through the National Research Foundation of Korea (NRF) funded by the Ministry of Education (NRF2015R1D1A1A01059467) and Hallym University Research Fund, 2014 (HRF-201409-017). Soon-Mo Jung was supported by Basic Science Research Program through the National Research Foundation of Korea (NRF) funded by the Ministry of Education (no. 2015R1D1A1A02061826).

\section{References}

[1] S. M. Ulam, A Collection of Mathematical Problems, Interscience, New York, NY, USA, 1960.

[2] D. H. Hyers, "On the stability of the linear functional equation," Proceedings of the National Academy of Sciences of the United States of America, vol. 27, pp. 222-224, 1941.

[3] S. Czerwik, Functional Equations and Inequalities in Several Variables, World Scientific, Singapore, 2002.
[4] P. Găvruta, "A generalization of the Hyers-Ulam-Rassias stability of approximately additive mappings," Journal of Mathematical Analysis and Applications, vol. 184, no. 3, pp. 431-436, 1994.

[5] D. H. Hyers, G. Isac, and Th. M. Rassias, Stability of Functional Equations in Several Variables, Birkhäuser, Boston, Mass, USA, 1998.

[6] S.-M. Jung, Hyers-Ulam-Rassias Stability of Functional Equations in Nonlinear Analysis, vol. 48 of Springer Optimization and Its Applications, Springer, New York, NY, USA, 2011.

[7] T. Aoki, "On the stability of the linear transformation in Banach spaces," Journal of the Mathematical Society of Japan, vol. 2, pp. 64-66, 1950.

[8] T. M. Rassias, "On the stability of the linear mapping in Banach spaces," Proceedings of the American Mathematical Society, vol. 72, no. 2, pp. 297-300, 1978.

[9] S.-M. Jung, "Approximation of analytic functions by special functions," Annals of Functional Analysis, vol. 3, no. 1, pp. 9299, 2012.

[10] M. Obloza, "Hyers stability of the linear differential equation," Rocznik Naukowo-Dydaktyczny, Prace Matematyczne, vol. 13, pp. 259-270, 1993.

[11] M. Obloza, "Connections between Hyers and Lyapunov stability of the ordinary differential equations," Rocznik NaukowoDydaktyczny. Prace Matematyczne, vol. 14, pp. 141-146, 1997.

[12] C. Alsina and R. Ger, "On some inequalities and stability results related to the exponential function," Journal of Inequalities and Applications, vol. 2, no. 4, pp. 373-380, 1998.

[13] T. Miura, S.-E. Takahasi, and H. Choda, "On the Hyers-Ulam stability of real continuous function valued differentiable map," Tokyo Journal of Mathematics, vol. 24, no. 2, pp. 467-476, 2001.

[14] T. Miura, S. Miyajima, and S.-E. Takahasi, "A characterization of Hyers-Ulam stability of first order linear differential operators," Journal of Mathematical Analysis and Applications, vol. 286, no. 1, pp. 136-146, 2003.

[15] S. E. Takahasi, T. Miura, and S. Miyajima, "On the Hyers-Ulam stability of the Banach space valued differential equation $y^{\prime}=$ $\lambda y$," Bulletin of the Korean Mathematical Society, vol. 39, pp. 309-315, 2002.

[16] S.-E. Takahasi, H. Takagi, T. Miura, and S. Miyajima, "The Hyers-Ulam stability constants of first order linear differential operators," Journal of Mathematical Analysis and Applications, vol. 296, no. 2, pp. 403-409, 2004

[17] S.-M. Jung, "Hyers-Ulam stability of linear differential equations of first order," Applied Mathematics Letters, vol. 17, no. 10, pp. 1135-1140, 2004.

[18] T. Miura, G. Hirasawa, and S. E. Takahasi, "Note on the HyersUlam-Rassias stability fo the first order linear differential equation $y(t)+p(t) y(t)+q(t)$," International Journal of Mathematics and Mathematical Sciences, vol. 22, pp. 1151-1158, 2004.

[19] G. Wang, M. Zhou, and L. Sun, "Hyers-Ulam stability of linear differential equations of first order," Applied Mathematics Letters, vol. 21, no. 10, pp. 1024-1028, 2008. 


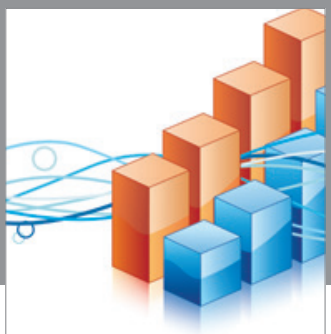

Advances in

Operations Research

vatem alat4

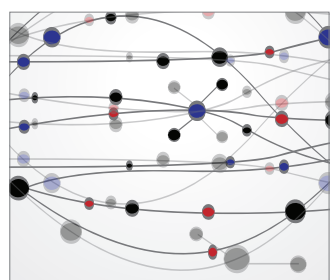

\section{The Scientific} World Journal
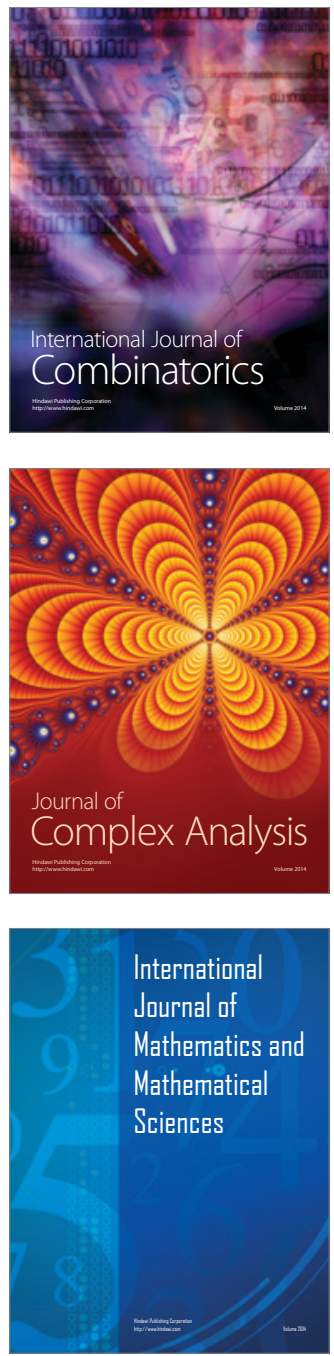
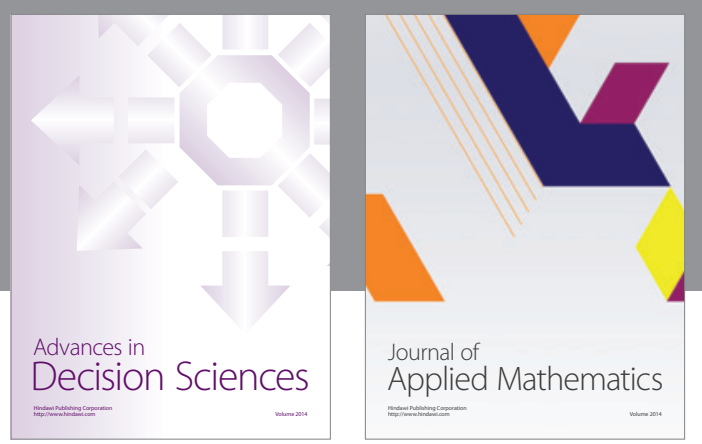

Algebra

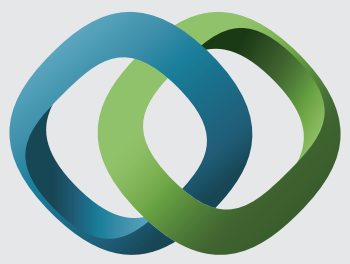

\section{Hindawi}

Submit your manuscripts at

http://www.hindawi.com
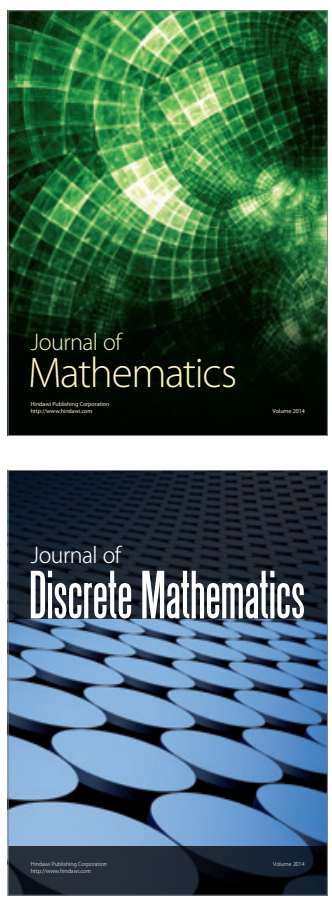

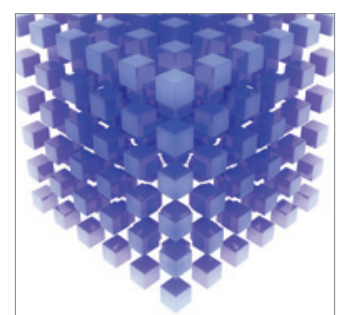

Mathematical Problems in Engineering
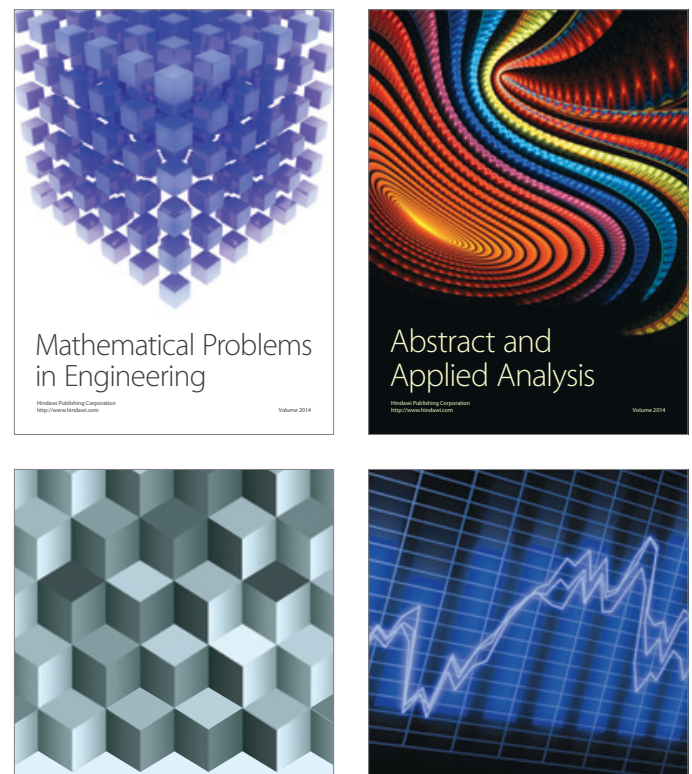

Journal of

Function Spaces

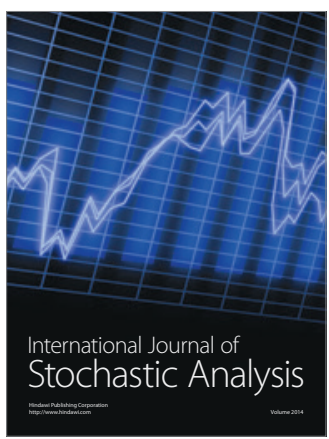

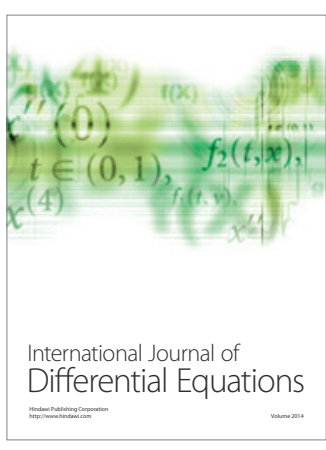
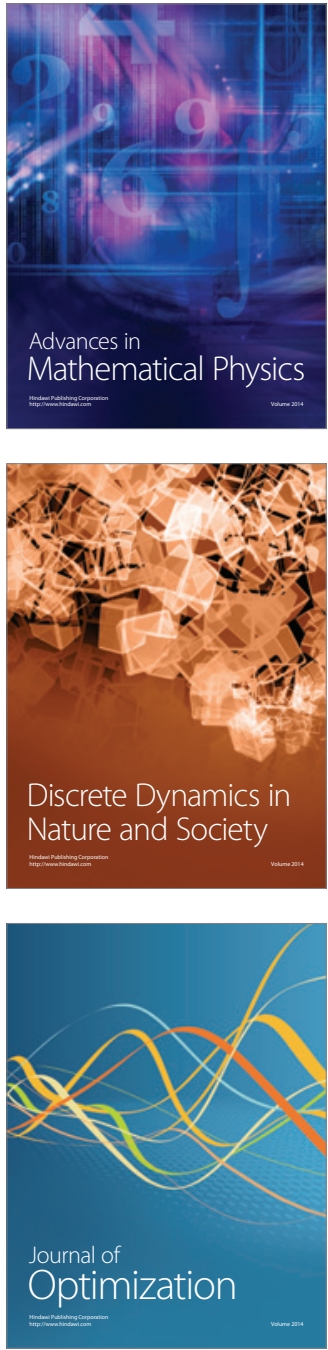